\title{
New agents in acute myeloid leukemia (AML)
}

\author{
Silvia Park ${ }^{1,2}$, Byung Sik Cho ${ }^{1,2}$, Hee-Je Kim ${ }^{1,2}$ \\ ${ }^{1}$ Department of Hematology, Catholic Hematology Hospital, Seoul St. Mary's Hospital, ${ }^{2}$ Leukemia Research Institute, College of \\ Medicine, The Catholic University of Korea, Seoul, Korea
}

p-ISSN 2287-979X / e-ISSN 2288-0011 https://doi.org/10.5045/br.2020.S003 Blood Res 2020;55:S14-S18.

Received on January 19, 2020

Revised on February 5, 2020

Accepted on February 18, 2020

\section{Correspondence to \\ Hee-Je Kim, M.D., Ph.D. \\ Department of Hematology, Catholic Hematology Hospital, Seoul St. Mary's Hospital, College of Medicine, The Catholic University of Korea, 222 Banpo-daero, Seocho-gu, Seoul 06591, Korea \\ E-mail: cumckim@catholic.ac.kr \\ (C) 2020 Korean Society of Hematology}

\begin{abstract}
Despite expanding knowledge in the molecular landscape of acute myeloid leukemia (AML) and an increasing understanding of leukemogenic pathways, little has changed in the treatment of AML in the last 40 years. Since introduction in the 1970s, combination chemotherapy consisting of anthracycline and cytarabine has been the mainstay of treatment, with major therapeutic advances based on improving supportive care rather than the introduction of novel therapeutics. Over the last decades, there have been extensive efforts to identify specific target mutations or pathways with the aim of improving clinical outcomes. Finally, after a prolonged wait, we are witnessing the next wave of AML treatment, characterized by a more "precise" and "personalized" understanding of the unique molecular or genetic mapping of individual patients. This new trend has since been further facilitated, with four new FDA approvals granted in 2017 in AML therapeutics. Currently, a total of eight targeted agents have been approved since 2017 (as of Jan. 2020). In this review, we will briefly discuss these newer agents in the context of their indication and the basis of their approval.
\end{abstract}

Key Words Acute myeloid leukemia, New FDA approvals

\section{INTRODUCTION}

Acute myeloid leukemia (AML) is a devasting disease, which rapidly becomes fatal if left untreated. Anthracycline and cytarabine-based combination chemotherapy has been the mainstay of treatment since its introduction in the 1970s $[1,2]$.

Recent advances in genomic technologies have identified that AML is a heterogeneous group of diseases, and the long-term prognosis of AML is significantly different and largely dependent on its own cytogenetics and molecular aberrations [3, 4]. Consequently, there have been substantial changes in the classification and prognostication of AML over the last few decades $[5,6]$, which are now predicated on identifying genetic features of the individual disease. Concurrently, efforts to discover potential molecular targets have been ongoing.

At the 2017 American Society of Hematology meeting, it was announced that 2017 was a landmark year for the Food and Drug Administration (FDA)-approved therapies for AML, as the U.S. FDA had approved four new therapeutic options for the disease (midostaurin, enasidenib, CPX-351, and gemtuzumab ozogamicin). Subsequently, four additional drugs (gilteritinib, ivosidenib, venetoclax, and glasdegib) have received U.S. FDA approval for AML [7]. Surprisingly, these advances have occurred only during the recent few years, and we are undoubtedly facing an "innovative era" in the treatment of AML.

In this review, we will focus on the recently approved new targeted agents for the treatment of AML.

\section{NEW AGENTS FOR AML WITH RECENT U.S. FDA APPROVAL}

Recently approved drugs for AML and their indication by disease status are listed in Table 1.

\section{MIDOSTAURIN}

In April 2017, midostaurin was approved for treatment of naïve FLT3-mutant AML, in combination with daunorubicin and cytarabine induction and cytarabine consolidation. Nearly $25-30 \%$ and $5-10 \%$ of patients with AML harbor an FLT3-internal tandem duplication (ITD) and -tyrosine kinase domain (TKD) mutation, respectively [8]. Among the 
Table 1. New agents for AML with recent U.S. FDA approval.

\begin{tabular}{|c|c|c|c|c|c|c|c|}
\hline & \multirow{2}{*}{$\begin{array}{l}\text { Molecular } \\
\text { target }\end{array}$} & \multicolumn{2}{|c|}{ Indication (setting) } & \multirow{2}{*}{ Representative trials } & \multirow{2}{*}{ OS benefit } & \multirow{2}{*}{$\begin{array}{c}\text { FDA } \\
\text { approval }\end{array}$} & \multirow{2}{*}{$\begin{array}{l}\text { In Korea } \\
\text { (Jan. 2020) }\end{array}$} \\
\hline & & New & $\mathrm{Rel} / \mathrm{ref}$ & & & & \\
\hline Midostaurin & FLT3 ITD/TKD & $\mathrm{O}\left(\mathrm{combi}^{\mathrm{a})}\right)$ & & Phase 3 RATIFY & Yes & Apr. 2017 & Available \\
\hline Gilteritinib & FLT3 ITD/TKD & & $\mathrm{O}$ (single) & Phase 3 ADMIRAL & Yes & Nov. 2018 & Not available \\
\hline $\begin{array}{l}\text { Gemtuzumab } \\
\text { ozogamicin } \\
\text { (GO) }\end{array}$ & CD33+ & $\begin{array}{l}\mathrm{O} \text { (combi } \\
\text { or single) }\end{array}$ & $\begin{array}{l}\mathrm{O} \text { (combi } \\
\text { or single) }\end{array}$ & $\begin{array}{l}\text { Phase } 3 \text { ALFA-0701 } \\
\text { Phase } 3 \text { AML-19 } \\
\text { Phase } 2 \text { MyloFrance-1 }\end{array}$ & $\begin{array}{c}\text { No (but benefit in EFS) } \\
\text { Yes } \\
\text { NA }\end{array}$ & Jul. 2017 & $\begin{array}{l}\text { Available } \\
\text { (via KOEDC) }\end{array}$ \\
\hline CPX-351 & $\begin{array}{l}\text { t-AML } \\
\text { AML-MRC }\end{array}$ & $\mathrm{O}$ & & Phase 3 (NCT01696084) & Yes & Aug. 2017 & Not available \\
\hline Enasidenib & $\mathrm{IDH} 2$ & & $\mathrm{O}$ (single) & $\begin{array}{l}\text { Phase } 1 / 2 \text { study } \\
\text { (NCT01915498) }\end{array}$ & NA & Aug. 2017 & Not available \\
\hline Ivosidenib & IDH1 & & $\mathrm{O}$ (single) & $\begin{array}{l}\text { Phase } 1 \text { dose-escalation/ } \\
\text { dose-expansion study } \\
\text { (NCT02074839) }\end{array}$ & NA & Jul. 2018 & Not available \\
\hline \multirow[t]{2}{*}{ Venetoclax } & $\mathrm{BCL} 2$ & $\mathrm{O}\left(\mathrm{combi}^{\mathrm{b})}\right)$ & & $\begin{array}{l}\text { Phase } 1 \text { dose-escalation } \\
\text { study (NCT02203773) }\end{array}$ & NA & Nov. 2018 & Available \\
\hline & & & & $\begin{array}{l}\text { Phase } 1 / 2 \text { study } \\
\text { (NCT02287233) }\end{array}$ & NA & & \\
\hline Glasdegib & $\begin{array}{l}\text { Hedgehog } \\
\text { signaling } \\
\text { pathway }\end{array}$ & $\mathrm{O}\left(\mathrm{combi}^{\mathrm{c})}\right)$ & & Phase 2 (NCT01546038) & Yes & Nov. 2018 & Not available \\
\hline
\end{tabular}

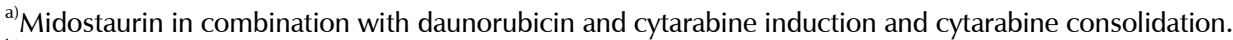

${ }^{b}$ Hypomethylating agents (HMA) such as decitabine or azacitidine with venetoclax (400 mg); low dose Ara-C (LDAC) with venetoclax (600 mg). ${ }^{c}$ Glasdegib and LDAC.

Abbreviations: BCL2, B-cell lymphoma 2; EFS, event-free survival; FLT3, FMS-like tyrosine kinase 3; IDH, isocitrate dehydrogenase; ITD, internal tandem duplication; KOEDC, Korea Orphan \& Essential Drug Center; NA, not assessable; OS, overall survival; rel/Ref, relapsed or refractory; $\mathrm{t}-\mathrm{AML}$, therapy-related $\mathrm{AML}$; $\mathrm{AML}-\mathrm{MRC}$, $\mathrm{AML}$ with myelodysplasia related changes; TKD, tyrosine kinase domain.

mutation locations, FLT3-ITD AML often presents hyperleukocytosis $[9,10]$ and is a known marker of adverse prognosis. However, the prognostic implication of FLT3TKD mutation remains unclear [11]. Based on the RATIFY trial (randomized AML trial in FLT3-mutated adults younger than 60 years old) [12, 13], in which 717 patients were randomly assigned to chemo $+/$ - midostaurin after screening approximately 3,300 patients, midostaurin was approved by the U.S. FDA after demonstrating a survival benefit in AML patients with mutated FLT3-ITD or TKD. This was the first FLT3 inhibitor approved in AML, breaking new ground for the trend of precision medicine in AML treatment.

\section{GILTERITINIB}

Gilteritinib, for use as a single agent, was approved for relapsed or refractory (R/R) FLT3-ITD and/or FLT3-TKD mutant AML in November 2018. As mentioned earlier, nearly one-third of AML patients have an activating mutation in FLT3. Given that the FLT3 mutation is frequently observed and strongly associated with relapse and survival in AML, many novel agents have attempted to treat AML harboring the FLT3 mutation [8, 11, 12]. Gilteritinib, a second-generation FLT3 inhibitor, is characterized by highly specific and potent inhibition of FLT3, with narrower kinome profiles than first-generation FLT3 inhibitors such as midostaurin $[14,15]$. The approval of this drug in AML was based on the phase 3 ADMIRAL trial [16], which demonstrated that gilteritinib significantly prolongs overall survival in FLT3 mutated relapsed/refractory AML when compared to comparative salvage chemotherapy arms.

\section{GEMTUZUMAB OZOGAMICIN}

In July 2017, gemtuzumab ozogamicin (GO), in combination with daunorubicin and cytarabine or as a single agent, was approved in adults for the treatment of newly diagnosed CD33-positive AML. Additionally, the FDA approved GO in $\mathrm{R} / \mathrm{R}$ CD33-positive AML in adults and pediatric patients at least two years of age. GO is a recombinant, humanized anti-CD-33 antibody conjugated to the intracellular toxin, calicheamicin [17]. In May 2000, this drug originally received accelerated approval for CD33-positive AML patients (60 $\mathrm{yr}$ or older) experiencing their first relapse, and in those not considered candidates for other cytotoxic chemotherapy [18]. Of note, Go combined with attenuated conventional chemotherapy was feasible in elderly patients with AML in Korea [19, 20]. Subsequently, in 2010, it was voluntarily withdrawn from the market due to failure to verify prior efficacy data, as well safety concerns, including a high number of early deaths and hepatic veno-occlusive disease (VOD). Interestingly, however, this drug has remained in the Japanese market based on an individual approach with Pfizer's compassionate use program, which reflects the critical unmet 
need for novel agents in AML treatment. Indeed, GO with different dosing and different schedules remained of great interest among AML clinicians, resulting in independent investigators conducting investigator-initiated clinical trials, including ALFA- 0701 (phase 3 study of GO with daunorubicin and cytarabine in 50-70 aged untreated AML) [21], AML-19 (phase 3 study of single-agent GO in newly diagnosed elderly AML) [22], and MyloFrance-1 (phase 2 open-label study of single-agent GO as induction therapy in relapsed AML) [23]. These studies formed the basis for the reapproval of $\mathrm{GO}$, demonstrating an improved event-free survival (EFS), overall survival (OS), or relapse-free survival (RFS). The current approval has been granted for a lower dose "fractionated" schedule rather than the dose originally approved by the FDA (2 doses of $9 \mathrm{mg} / \mathrm{m}^{2}$ each on days 1 and 8), aimed at a different target population. Currently, the widely accepted protocol of GO is $3 \mathrm{mg} / \mathrm{m}^{2}$ on days 1,3 , and 5; this reduced and fractionated dose schedule seems to increase tolerance and decrease drug-related toxicity such as VOD [24, 25].

\section{CPX-351}

In August 2017, CPX-351 was approved for newly diagnosed therapy-related AML (t-AML) or AML with myelodysplasia-related changes (AML-MRC). CPX-351 is a liposomal formulation that delivers a 5:1 fixed-molar ratio of daunorubicin and cytarabine [26, 27]. The approval was based on results of a phase 3 clinical trial in 309 patients with either t-AML or AML-MRC, which evaluated the efficacy and safety of CPX-351 compared to conventional cytarabine and daunorubicin induction [28]. In this study, CPX-351 demonstrated an improvement in OS, with a median OS of 9.56 months for the CPX-351 arm versus 5.95 months for the conventional chemotherapy arm. Although the trial was conducted only in patients aged 60 to 75 years, the FDA approved CPX-351 for use in all adults with no age restrictions.

\section{ENASIDENIB AND IVOSIDENIB}

Enasidenib, for use as a single agent, was approved by the FDA in August 2017 for R/R AML with the isocitrate dehydrogenase-2 (IDH2) mutation. In July 2018, ivosidenib was also approved as a single agent in $I D H 1$-mutated R/R AML. Enasidenib [29-31] and ivosidenib [32-34] are small molecule inhibitors targeting $I D H 2$ and $I D H 1$, respectively. Isocitrate dehydrogenase (IDH) plays a key role in converting isocitrate to $\alpha$-ketoglutarate $(\alpha-K G)$, demonstrating the three different isoforms of IDH1, 2 and 3 . The $I D H$ mutation results in a neomorphic enzyme, which can result in the abnormal accumulation of the oncometabolite R-2-hydroxyglutarate (R-2-HG) and promote leukemogenesis [35]. Notably, nearly $6-10 \%$ of AML patients bear a mutation in $I D H 1$, and mutations in $I D H 2$ are observed in $9-13 \%$ of AML patients [36-38]. However, reports on the prognostic potential of $I D H 1$ and $I D H 2$ mutations have been inconsistent. The approval of enasidenib in AML was based on a phase $1 / 2$ study [31]. In this study, patients with $I D H 2$ mutated $\mathrm{R} / \mathrm{R}$ AML, treated with single-agent enasidenib, demonstrated a response rate of $40.3 \%$ [complete remission (CR) rate of $19.3 \%$ ], and median OS of 9.3 months. Similarly, ivosidenib was approved based on a large phase 1 dose-escalation/dose-expansion trial [32]. In this study, IDH1 mutated R/R AML patients treated with single-agent ivosidenib demonstrated a response rate of $41.6 \%$ [composite CR (cCR) rate of $30.4 \%$ ] and median OS of 8.8 months. As an adverse event of special interest, differentiation syndrome was observed in patients treated with enasidenib (7\%) and ivosidenib (3.9\%). Reportedly, the IDH-differentiation syndrome is manageable by interrupting medication and treatment with glucocorticoids, with or without hydroxyurea [39, 40].

\section{VENETOCLAX}

Venetoclax, in combination with azacitidine or decitabine or low dose cytarabine, received accelerated FDA approval in Nov 2018 for newly diagnosed AML (age 75 $\geq$ yr or "unfit" patients). Venetoclax is an orally active, and potent small molecule inhibitor targeting B-cell lymphoma 2 (BCL2) [41]. The approval of this new agent was based on two open-label non-randomized trials of Study M14-358 (NCT02203773) [42] and Study M14-387 (NCT02287233) [43]. In M14-358 (Phase 1 dose-escalation study), venetoclax in combination with azacitidine or decitabine was evaluated in newly diagnosed elderly ( $\geq 65 \mathrm{yr}$ ), unfit AML patients, with venetoclax doses of 400,800 , or $1,200 \mathrm{mg}$ daily. The median age of enrolled patients was 74 years, and the poor-risk cytogenetics comprised $49 \%$. Promising results were observed with cCRs of $67 \%$ (all doses) and 73\% (venetoclax $400 \mathrm{mg}$ ), the median duration of cCR was 11.3 months and the median OS was 17.5 months. Unexpectedly, the results demonstrated that patients with poor-risk cytogenetics and those at least 75 years of age reported cCR rates of $60 \%$ and $65 \%$, respectively. Additionally, M14-387 (NCT02287233) was another notable study that contributed to the accelerated approval of venetoclax. This phase I/II study evaluated the safety and preliminary efficacy of venetoclax $(600 \mathrm{mg})$ with low dose cytarabine (LDAC) in untreated elderly ( $\geq 60 \mathrm{yr}$ ) or unfit AML patients. The median age of enrolled patients was 74 years and the poor-risk cytogenetics comprised 32\%. Nearly one-third of patients $(29 \%)$ had received prior hypomethylating agents (HMA) treatment. In this difficult-to-treat population, 54\% achieved cCR, median OS was 10.1 months, and the median response duration was 8.1 months. Based on the aforementioned trials $[42,43]$, the recommended venetoclax dose depends on the combination regimen: 400 mg daily in combination with HMA and $600 \mathrm{mg}$ daily in combination with LDAC [7]. 


\section{GLASDEGIB}

Glasdegib, in combination with LDAC, was approved by the FDA in November 2018 for the frontline treatment of elderly ( $>75 \mathrm{yr}$ ) or unfit patients. Glasdegib is an oral inhibitor of the hedgehog signaling pathway $[44,45]$. Recently, the aberrant activation of the hedgehog signaling pathway has been implicated in the maintenance and development of several malignancies including AML [46]. Glasdegib received FDA approval based on a phase II, randomized, open-label, multicenter study (NCT01546038), in which 88 and 44 patients (older than $75 \mathrm{yr}$ or older than $55 \mathrm{yr}$ with significant comorbidities) were randomized to glasdegib/ LDAC and LDAC, respectively [47]. The results demonstrated that glasdegib/LDAC was superior to LDAC alone, indicating better survival (the median OS of 8.3 mo vs. 4.9 mo, $P=0.0004)$ and a higher $\mathrm{CR}$ rate $(17.0 \%$ vs. $2.3 \%, P<0.05)$ in the glasdegib/LDAC combination arm.

\section{CONCLUSION AND DISCUSSION}

Over the years, there has been a gradual paradigm shift from traditional medicine to personalized or precision medicine owing to the explosive growth in available genetic data [48]. Traditional or conventional medicine is characterized by empirical and mechanism-based treatments, targeting an entire population. On the contrary, precision medicine targets an individual patient based on the understanding of the unique molecular mechanisms of the patient $[48,49]$.

In the field of AML, there has been the growing understanding and discovery of leukemogenic pathways in the past years, and an innovative approach to AML treatment has "long" been expected. However, in the last 40 years, little has changed in the treatment of AML. Moreover, the majority of therapeutic advances in AML are associated with improved supportive care rather than the introduction of novel therapeutics.

Fortunately, with the introduction of numerous novel agents, we are now witnessing a new era in AML treatment [7]. Notably, 2017 was a memorable year in the history of AML treatment, marking the success of biomedical research efforts over the last several decades. The one of future challenges will be to incorporate and use these new therapeutics where they have the greatest impact - use alone or combine targeted agents with each other or with conventional chemotherapy [50].

Lastly, in Korea, we still have a long way to go, with limited access to these novel agents in clinical practice. Although these are not all-around, I hope that these new FDA approvals are no longer 'a pie in the sky' for Korean patients as well in a near future.

\section{Authors' Disclosures of Potential Conflicts of Interest}

No potential conflicts of interest relevant to this article were reported.

\section{REFERENCES}

1. Jang J, Lee J, Jang JH, Jung CW, Park S. Anti-leukemic effects of simvastatin on NRAS(G12D) mutant acute myeloid leukemia cells. Mol Biol Rep 2019;46:5859-66.

2. Roboz GJ. Novel approaches to the treatment of acute myeloid leukemia. Hematology Am Soc Hematol Educ Program 2011; 2011:43-50.

3. Bullinger L, Döhner K, Döhner H. Genomics of acute myeloid leukemia diagnosis and pathways. J Clin Oncol 2017;35:934-46.

4. Papaemmanuil E, Gerstung M, Bullinger L, et al. Genomic classification and prognosis in acute myeloid Leukemia. N Engl J Med 2016;374:2209-21.

5. Arber DA, Orazi A, Hasserjian R, et al. The 2016 revision to the World Health Organization classification of myeloid neoplasms and acute leukemia. Blood 2016;127:2391-405.

6. Leonard JP, Martin P, Roboz GJ. Practical implications of the 2016 revision of the World Health Organization classification of lymphoid and myeloid neoplasms and acute leukemia. J Clin Oncol 2017;35:2708-15.

7. Kopmar NE, Estey EH. New drug approvals in acute myeloid leukemia: an unprecedented paradigm shift. Clin Adv Hematol Oncol 2019;17:569-75.

8. Kazi JU, Rönnstrand L. FMS-like tyrosine kinase 3/FLT3: from basic science to clinical implications. Physiol Rev 2019;99: 1433-66.

9. Canaani J, Labopin M, Socié G, et al. Long term impact of hyperleukocytosis in newly diagnosed acute myeloid leukemia patients undergoing allogeneic stem cell transplantation: an analysis from the acute leukemia working party of the EBMT. Am J Hematol 2017;92:653-9.

10. Tien FM, Hou HA, Tsai CH, et al. Hyperleukocytosis is associated with distinct genetic alterations and is an independent poor-risk factor in de novo acute myeloid leukemia patients. Eur J Haematol 2018;101:86-94.

11. Short NJ, Kantarjian H, Ravandi F, Daver N. Emerging treatment paradigms with FLT3 inhibitors in acute myeloid leukemia. Ther Adv Hematol 2019;10:2040620719827310.

12. Levis M. Midostaurin approved for FLT3-mutated AML. Blood 2017;129:3403-6.

13. Stone RM, Mandrekar SJ, Sanford BL, et al. Midostaurin plus chemotherapy for acute myeloid leukemia with a FLT3 mutation. N Engl J Med 2017;377:454-64.

14. Daver N, Schlenk RF, Russell NH, Levis MJ. Targeting FLT3 mutations in AML: review of current knowledge and evidence. Leukemia 2019;33:299-312.

15. Larrosa-Garcia M, Baer MR. FLT3 inhibitors in acute myeloid leukemia: current status and future directions. Mol Cancer Ther 2017;16:991-1001.

16. Perl AE, Martinelli G, Cortes JE, et al. Gilteritinib or chemotherapy 
for relapsed or refractory FLT3-mutated AML. N Engl J Med 2019;381:1728-40.

17. Godwin CD, Gale RP, Walter RB. Gemtuzumab ozogamicin in acute myeloid leukemia. Leukemia 2017;31:1855-68.

18. Bross PF, Beitz J, Chen G, et al. Approval summary: gemtuzumab ozogamicin in relapsed acute myeloid leukemia. Clin Cancer Res 2001;7:1490-6.

19. Eom KS, Kim HJ, Min WS, et al. Gemtuzumab ozogamicin in combination with attenuated doses of standard induction chemotherapy can successfully induce complete remission without increasing toxicity in patients with acute myeloid leukemia aged 55 or older. Eur J Haematol 2007;79:398-404.

20. Eom KS, Kim HJ, Cho BS, et al. Hematopoietic stem cell transplant following remission induction chemotherapy including gemtuzumab ozogamicin is a feasible and effective treatment option in elderly patients with acute myeloid leukemia. Leuk Lymphoma 2011;52:2321-8.

21. Castaigne $S$, Pautas $C$, Terré $C$, et al. Effect of gemtuzumab ozogamicin on survival of adult patients with de-novo acute myeloid leukaemia (ALFA-0701): a randomised, open-label, phase 3 study. Lancet 2012;379:1508-16.

22. Amadori S, Suciu S, Selleslag D, et al. Gemtuzumab ozogamicin versus best supportive care in older patients with newly diagnosed acute myeloid leukemia unsuitable for intensive chemotherapy: results of the randomized phase III EORTC-GIMEMA AML-19 Trial. J Clin Oncol 2016;34:972-9.

23. Taksin AL, Legrand O, Raffoux E, et al. High efficacy and safety profile of fractionated doses of Mylotarg as induction therapy in patients with relapsed acute myeloblastic leukemia: a prospective study of the alfa group. Leukemia 2007;21:66-71.

24. Baron J, Wang ES. Gemtuzumab ozogamicin for the treatment of acute myeloid leukemia. Expert Rev Clin Pharmacol 2018;11:549-59.

25. Yu B, Liu D. Gemtuzumab ozogamicin and novel antibody-drug conjugates in clinical trials for acute myeloid leukemia. Biomark Res 2019;7:24

26. Mayer LD, Tardi P, Louie AC. CPX-351: a nanoscale liposomal co-formulation of daunorubicin and cytarabine with unique biodistribution and tumor cell uptake properties. Int J Nanomedicine 2019;14:3819-30.

27. Feldman EJ, Kolitz JE, Trang JM, et al. Pharmacokinetics of CPX-351; a nano-scale liposomal fixed molar ratio formulation of cytarabine:daunorubicin, in patients with advanced leukemia. Leuk Res 2012;36:1283-9.

28. Lancet JE, Uy GL, Cortes JE, et al. CPX-351 (cytarabine and daunorubicin) liposome for injection versus conventional cytarabine plus daunorubicin in older patients with newly diagnosed secondary acute myeloid leukemia. J Clin Oncol 2018;36:2684-92.

29. Dogra R, Bhatia R, Shankar R, Bansal P, Rawal RK. Enasidenib: first mutant IDH2 inhibitor for the treatment of refractory and relapsed acute myeloid leukemia. Anticancer Agents Med Chem 2018;18:1936-51.

30. Stein EM. Enasidenib, a targeted inhibitor of mutant IDH2 proteins for treatment of relapsed or refractory acute myeloid leukemia. Future Oncol 2018;14:23-40.

31. Stein EM, DiNardo CD, Pollyea DA, et al. Enasidenib in mutant IDH2 relapsed or refractory acute myeloid leukemia. Blood 2017;130:722-31.
32. DiNardo CD, Stein EM, de Botton S, et al. Durable remissions with ivosidenib in IDH1-mutated relapsed or refractory AML. N Engl J Med 2018;378:2386-98.

33. Roboz GJ, DiNardo CD, Stein EM, et al. Ivosidenib induces deep durable remissions in patients with newly diagnosed IDH1mutant acute myeloid leukemia. Blood 2019. [Epub ahead of print]

34. Sharma H. Development of novel therapeutics targeting isocitrate dehydrogenase mutations in cancer. Curr Top Med Chem 2018; 18:505-24.

35. Montalban-Bravo G, DiNardo CD. The role of IDH mutations in acute myeloid leukemia. Future Oncol 2018;14:979-93.

36. Medeiros BC, Fathi AT, DiNardo CD, Pollyea DA, Chan SM, Swords R. Isocitrate dehydrogenase mutations in myeloid malignancies. Leukemia 2017;31:272-81.

37. Schnittger S, Haferlach C, Ulke M, Alpermann T, Kern W, Haferlach T. IDH1 mutations are detected in $6.6 \%$ of 1414 AML patients and are associated with intermediate risk karyotype and unfavorable prognosis in adults younger than 60 years and unmutated NPM1 status. Blood 2010;116:5486-96.

38. Wouters BJ. Hitting the target in IDH2 mutant AML. Blood 2017;130:693-4.

39. Fathi AT, DiNardo CD, Kline I, et al. Differentiation syndrome associated with enasidenib, a selective inhibitor of mutant isocitrate dehydrogenase 2 : analysis of a phase $1 / 2$ study. JAMA Oncol 2018;4:1106-10.

40. Megías-Vericat JE, Ballesta-López O, Barragán E, Montesinos P. IDH1-mutated relapsed or refractory AML: current challenges and future prospects. Blood Lymphat Cancer 2019;9:19-32.

41. Juárez-Salcedo LM, Desai V, Dalia S. Venetoclax: evidence to date and clinical potential. Drugs Context 2019;8:212574.

42. DiNardo CD, Pratz K, Pullarkat V, et al. Venetoclax combined with decitabine or azacitidine in treatment-naive, elderly patients with acute myeloid leukemia. Blood 2019;133:7-17.

43. Wei AH, Strickland SA Jr, Hou JZ, et al. Venetoclax combined with low-dose cytarabine for previously untreated patients with acute myeloid leukemia: results from a phase Ib/II study. J Clin Oncol 2019;37:1277-84.

44. Goldsmith SR, Lovell AR, Schroeder MA. Glasdegib for the treatment of adult patients with newly diagnosed acute myeloid leukemia or high-grade myelodysplastic syndrome who are elderly or otherwise unfit for standard induction chemotherapy. Drugs Today (Barc) 2019;55:545-62.

45. Wolska-Washer A, Robak T. Glasdegib in the treatment of acute myeloid leukemia. Future Oncol 2019;15:3219-32.

46. Terao T, Minami Y. Targeting hedgehog (Hh) pathway for the acute myeloid leukemia treatment. Cells 2019;8:312.

47. Cortes JE, Heidel FH, Hellmann A, et al. Randomized comparison of low dose cytarabine with or without glasdegib in patients with newly diagnosed acute myeloid leukemia or high-risk myelodysplastic syndrome. Leukemia 2019;33:379-89.

48. Agyeman AA, Ofori-Asenso R. Perspective: does personalized medicine hold the future for medicine? J Pharm Bioallied Sci 2015;7:239-44.

49. Goldberger JJ, Buxton AE. Personalized medicine vs guidelinebased medicine. JAMA 2013;309:2559-60.

50. Rowe JM. Will new agents impact survival in AML? Best Pract Res Clin Haematol 2019;32:101094 